\title{
Mono- and bi-allelic effects of coding variants on disease in 176,899 Finns
}

H. O. Heyne $e^{1-5^{*}}$, J. Karjalainen ${ }^{1,5-8}$, K. J. Karczewski ${ }^{1,5-8}$, S. M. Lemmelä ${ }^{1,9}$, W. 8,10, M. J. Daly ${ }^{1,5-8^{*}}$

1 Finnish Institute for Molecular Medicine (FIMM), University of Helsinki, Helsinki, Finland

2 Digital Health Center, Hasso Plattner Institute for Digital Engineering, University of

10 Potsdam, Potsdam, Germany

113 Hasso Plattner Institute for Digital Health at Mount Sinai, Icahn School of Medicine at Mount

12 Sinai, New York, NY, USA

134 Department of Genetics and Genomic Sciences, Icahn School of Medicine at Mount Sinai, New

14 York, NY, USA

155 Program for Medical and Population Genetics, Broad Institute of MIT and Harvard, Cambridge,

16 MA, USA

176 Stanley Center for Psychiatric Research, Broad Institute of MIT and Harvard, Cambridge, MA,

18 USA

197 Analytic and Translational Genetics Unit, Massachusetts General Hospital, Boston, MA, USA

208 Center for Genomic Medicine, Massachusetts General Hospital, Boston, MA, USA

219 Finnish Institute for Health and Welfare, Helsinki, Finland

2210 Psychiatric \& Neurodevelopmental Genetics Unit, Department of Psychiatry, Massachusetts

23 General Hospital, Boston, Massachusetts, United States of America

*corresponding authors

26 henrike.heyne@hpi.de

27 mark.daly@helsinki.fi

28

30 Fl-00014 University of Helsinki

31 FINLAND 
medRxiv preprint doi: https://doi.org/10.1101/2021.11.06.21265920; this version posted November 11, 2021. The copyright holder for this preprint (which was not certified by peer review) is the author/funder, who has granted medRxiv a license to display the preprint in It is made available under a CC-BY 4.0 International license.

\section{Abstract}

34 Identifying Mendelian diseases with recessive inheritance is challenging as the majority of 35 cases are caused by compound heterozygous genotypes which require sequencing data 36 in families to definitively identify. Bottleneck events, such as in the Finnish population, 37 enrich specific homozygous variants to higher frequencies and thus facilitate identification 38 of disease associations through easily recognized homozygous genotypes. Here, we study 39 homozygous and heterozygous effects of 82,516 coding variants on 2,444 disease 40 endpoints using nationwide electronic health record (EHR) data of 176,899 Finns. We find 41 known and novel associations to homozygous genotypes across a broad spectrum of 42 phenotypes such as retinal dystrophy, adult-onset cataract and female infertility (13/20 of 43 which would have been missed by the traditional additive GWAS model). With these 44 results, and supporting simulations, we demonstrate the added benefit of homozygous 45 scans in GWAS. We further use these results to explore inheritance patterns of known 46 Mendelian variants. We find many Mendelian variants whose inheritance cannot be 47 adequately described with the traditional definition of dominant or recessive. In particular, 48 we find disease risk in heterozygous carriers of variants known to cause disease with recessive inheritance, as well as for variants labeled benign in ClinVar. Our results demonstrate how biobank efforts, particularly in founder populations, can broaden our understanding of the impact of genetic variants. 
medRxiv preprint doi: https://doi.org/10.1101/2021.11.06.21265920; this version posted November 11, 2021. The copyright holder for this preprint (which was not certified by peer review) is the author/funder, who has granted medRxiv a license to display the preprint in perpetuity.

It is made available under a CC-BY 4.0 International license .

\section{Main}

Introduction.

Rare genetic variants with large effects on disease can have potential direct treatment implications ${ }^{1,2}$; studying their effects comprehensively requires however large sample sizes $^{3}$. Identifying new bi-allelic effects on disease is particularly challenging, as the majority of causal variants with autosomal recessive inheritance are compound heterozygous in most individuals from non-isolated and non-consanguineous populations $\mathbf{s}^{4}$, owing to selective pressure keeping individual variants at low frequency. However, this is not the case in populations that have undergone bottleneck events such as Ashkenazi Jews $^{5}$, Icelanders ${ }^{6}$, French Canadians ${ }^{7}$, Anabaptist groups ${ }^{8}$, Puerto Ricans ${ }^{9}$ or Finns ${ }^{10}$. The Finnish population has been small and relatively isolated with lower genetic diversity than other European populations ${ }^{11}$. Multiple bottleneck events in the Finnish population history can today be detected as geographically distinct genetic clusters that partially correspond to historical events and local dialects ${ }^{12,13}$. Such geographically distinct genetic clusters are also characterized by higher rates of haplotype sharing ${ }^{12,14}$ and thus, higher rates of homozygosity increasing the chance occurrence of pathogenic variants in a homozygous state that lead to disease with recessive inheritance. This results in an enrichment of specific Mendelian genetic diseases such as congenital nephrotic syndrome (Finnish type $)^{15}$ or Northern epilepsy syndrome ${ }^{16}$ in certain areas of Finland today. Collectively, these currently 36 "founder diseases" are referred to as the "Finnish disease heritage" 10,17 and show mostly autosomal recessive inheritance. Populations that underwent recent bottlenecks are also characterized by an enrichment of mildly deleterious variants, including variants causing disease with recessive inheritance, as those stochastically rose in frequency following a bottleneck event but did not yet drop to the lower levels by purging as in long-term large populations ${ }^{3}$. In line with that, potentially deleterious variant classes (such as protein truncating point mutations) are specifically enriched at an intermediate frequency range (i.e. ca. $0.5 \%-5 \%$ ) in Finland ${ }^{18,19}$. An enrichment of founder diseases with recessive inheritance has also been observed in other isolated populations such as Tay Sachs or Gaucher in Ashkenazi Jews ${ }^{20}$, HermanskyPudlak syndrome in Puerto Ricans ${ }^{21}$ or ARSACS in French Canadians ${ }^{22}$. Isolated populations have been successfully used to map disease genes for decades ${ }^{6,11,23}$ as the higher allele frequencies of founder variants increases statistical power for detecting disease associations and long shared haplotypes across families facilitated linkage disequilibrium mapping at lower map resolution. While Mendelian variants of high penetrance can segregate and thus be identified in family studies in non-bottlenecked populations, family studies have not proven ideal for finding disease genes in more 
medRxiv preprint doi: https://doi.org/10.1101/2021.11.06.21265920; this version posted November 11, 2021. The copyright holder for this preprint (which was not certified by peer review) is the author/funder, who has granted medRxiv a license to display the preprint in perpetuity.

It is made available under a CC-BY 4.0 International license.

complex and common diseases ${ }^{3}$ given the massive polygenicity and incomplete penetrance which demand profoundly larger sample sizes to support discovery. An additional power advantage when doing case control studies in isolated populations comes from the reduced variant diversity, which decreases noise from neutral genetic variants and also helps the identification of causal disease variants e.g. Finns have only $1 / 3$ ultrarare truncating variants as Non-Finnish Europeans ${ }^{19}$. On the other hand, statistical finemapping to identify causal variants ${ }^{24}$ may be more difficult in populations with recent bottlenecks due to longer extent of linkage disequilibrium. Some relatively common European Mendelian disease variants in e.g. CFTR causing cystic fibrosis ${ }^{25}$ or $B R C A 1 / B R C A 2$ causing breast cancer are at much lower frequencies in Finland. On the other hand, greater haplotype sharing in the Finnish population facilitates imputation of lower frequency variants in array data from deep whole genome sequencing data with a population-specific reference panel down to frequencies below $0.0005^{26}$. Genotypes can thus be generated with arrays thereby enabling the large scale of the FinnGen research project. Its participants are largely recruited via hospital biobanks and are enriched for individuals with diseases across the clinical spectrum. The phenotypes are derived from national healthcare registry data collected over more than 50 years.

In this manuscript, we analyze the effects of 82,516 coding variants on 2,444 disease endpoints using nationwide EHR data of 176,899 Finns (FinnGen data freeze 4, 08/2019). In addition to the standard additive GWAS model ${ }^{27}$, we systematically search for homozygous effects. This enables us to explore two related questions of interest to both Mendelian and quantitative genetics communities. Firstly, we investigate the potential benefit of searching for homozygous effects in GWAS. Secondly, we explore the broad phenotypic consequences of mono- versus bi-allelic states of rare coding variants previously documented with a specific mode of inheritance in Mendelian disease.

Variants causing disease with recessive inheritance are at higher MAF in Finnish than Non-Finnish Europeans.

In light of a global enrichment of deleterious variants in Finland ${ }^{18,19}$ and the well-described "Finnish Disease Heritage"10,17, we hypothesized that a set of known disease-causing variants should exist at higher frequencies in Finland. We thus compared minor allele frequencies (MAF) of 2419 unique variants that were listed in the disease variant database $\mathrm{ClinVar}^{28}$ (version 2018) as pathogenic or likely pathogenic (=P/LP) in genes with only recessive inheritance annotated in the Online Mendelian Inheritance in Man, OMIM ${ }^{\circledR}$ (omim.org) between 10,824 Finnish (FIN) and 10,824 Non-Finnish Europeans (NFE) taken from the gnomAD population database ${ }^{29}$ (Figure 1). Concordant with a known lower rare variant diversity ${ }^{10}$, only 554 P/LP variants were present in FIN while 2169 were present in 
medRxiv preprint doi: https://doi.org/10.1101/2021.11.06.21265920; this version posted November 11, 2021. The copyright holder for this preprint (which was not certified by peer review) is the author/funder, who has granted medRxiv a license to display the preprint in

127 NFE at same sample sizes. As expected, however, observed P/LP variants were at higher

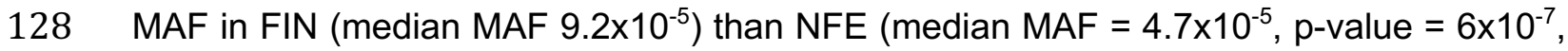
129 Wilcoxon rank test), with the difference particularly pronounced in 133 variants in Finnish 130 disease heritage genes ${ }^{17}$ (see Supplementary Figure S1). In FIN, 12\% (67/554) of P/LP 131 variants were above MAF 0.001 (39\% [217/554] above MAF $\left.10^{-4}\right)$ compared to $2.5 \%$ 132 (54/2169) in NFE (26\% [559/2169] above MAF 10-4).

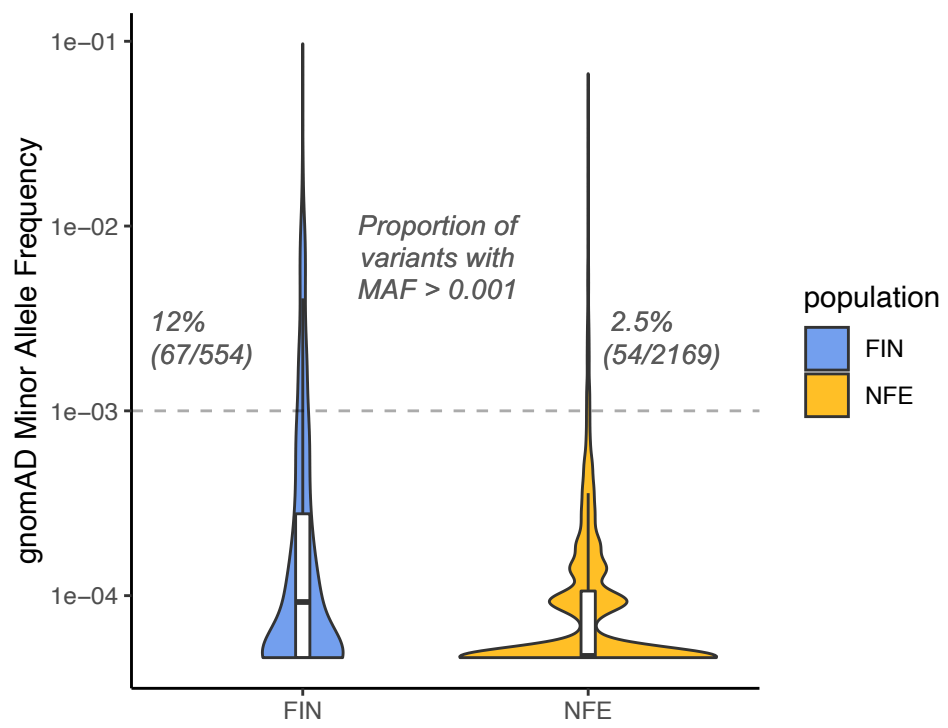

134 Figure 1. Variants known to cause disease with recessive inheritance are at higher 135 MAF in Finnish than non-Finnish Europeans. We show MAF of 2419 unique P/LP variants (source: ClinVar $^{28}$ ) in 10,824 individuals from FIN and 10,824 from NFE populations (source: gnomAD ${ }^{29}$ ). Violin plots are scaled to have the same area. Boxplots within violins show the 1st, 2nd and 3rd quartiles of the MAF distribution; whiskers maximally extend to 1.5 interquartile range.

141 Disease effects of variants known to (not) cause disease in 176, 899 Finns

142 With such a large fraction of these alleles common enough to explore in genotype-based 143 biobank studies, we examined 15,140 variants from ClinVar ${ }^{28}$ in 176,899 Finns from the 144 FinnGen research project. These included 311 coding variants that were annotated as 145 P/LP. We replicate known associations of the most frequent P/LP variants largely as 146 expected (see Supplementary Note 1, Supplementary Figure S2), demonstrating such 147 studies are powered to find disease associations across a wide range of phenotypes and 148 inheritance models - noting as examples variants in CHEK2, JAK2, OCA2 and MC1R that 149 showed semidominant effects in different cancer phenotypes (Supplementary Figure S2 $150 \mathrm{~F}, \mathrm{H}, \mathrm{I}, \mathrm{V}, \mathrm{W}, \mathrm{AC}, \mathrm{AD}, \mathrm{AE})$. In addition, we provide examples where these data can help verify 151 or falsify previously described disease associations. We provide longitudinal disease onset 
medRxiv preprint doi: https://doi.org/10.1101/2021.11.06.21265920; this version posted November 11, 2021. The copyright holder for this preprint (which was not certified by peer review) is the author/funder, who has granted medRxiv a license to display the preprint in perpetuity.

It is made available under a CC-BY 4.0 International license .

152 data of 26 variants, labeled as P/LP in ClinVar by at least one submitter, with genome153 wide significant associations in FinnGen (Supplementary Figure S2). We then investigated 154 global effects of known ClinVar variants in a phenome-wide association analysis 155 (pheWAS) of 2,444 disease phenotypes derived from health registry data using SAiGE ${ }^{27}$ 156 cognizant of the fact that for many rare variants we may only be powered to identify their 157 disease effects with moderate and not genome-wide levels of significance. To characterize 158 the broader impact of these variants, we compared the ClinVar variants to randomly 159 sampled intergenic variants in $15 \mathrm{MAF}$ bins and in the same $3 \mathrm{Mb}$ windows using different $160 \mathrm{p}$-value thresholds. As anticipated, we found significantly more phenotype associations 161 than expected at all $p$-value thresholds for variants that were labeled as P/LP in ClinVar in 162 genes described to cause disease with dominant inheritance (classification: OMIM). We

163 also found a global association with disease phenotypes for variants listed as benign or 164 likely benign (B/LB) in ClinVar regarded as "not implicated in monogenic disease" 30 and 165 often considered neutral ${ }^{31} .16 \mathrm{~B} / \mathrm{LB}$ variants were even the most probable causal SNP of 166 a GWAS locus following statistical finemapping ${ }^{24}$ (see Table 1). The ClinVar annotation 167 labeling these variants as B/LB was above average quality $(1.6 ; 7$ of the $16 \mathrm{~B} / \mathrm{LB}$ top causal 168 variants had a one star and 9 of 16 a two star review status in ClinVar) compared to an 169 average 1.2 stars for all B/LB variants in ClinVar (range: zero to three stars). As possible 170 explanations for these phenotype associations, we found, that B/LB variants have high 171 MAF, are more often protective and are more likely disease-associated than random 172 coding variants as they are defined to be in genes that were already associated with 173 disease (see Supplementary Note 2). An example is a protective B/LB probably causal 174 (posterior inclusion probability [PIP] 0.99) variant we observed in the gene $D B H$. $D B H$ is a 175 gene associated with dopamine beta hydroxylase deficiency with recessive inheritance.

176 This disease is characterized by severe hypotension ${ }^{32}$. We observed a missense variant 177 in $D B H$ that conveys protection from hypertension ( $\mathrm{p}$-value $5 \times 10^{-13}$, beta -0.17 , see Figure $1783 \mathrm{~A}$ ), a plausible finding given $D B H$ 's association with hypotension. The variant is also an 179 example for a Finnish enriched variant (22 times higher AF than in non Finnish-Swedish180 Estonian Europeans with an AF 0.05 in Finland).

Heterozygous effects of variants with known recessive inheritance

183 We found a global phenotype association signal for P/LP variants in disease genes with 184 recessive inheritance (see Supplementary Figure S3). This could suggest some variants with recessive inheritance may have disease effects in a heterozygous state or simply that 186 the additive model is detecting their known homozygous effects. To distinguish these 187 possibilities, we set out to explore if heterozygous effects contributed to the global 188 phenotype associations of P/LP variants in genes with recessive inheritance. First, we 
medRxiv preprint doi: https://doi.org/10.1101/2021.11.06.21265920; this version posted November 11, 2021. The copyright holder for this preprint (which was not certified by peer review) is the author/funder, who has granted medRxiv a license to display the preprint in perpetuity.

It is made available under a CC-BY 4.0 International license.

performed simulations of genotype and phenotype data (see Supplementary Note 3) and compared how well additive and recessive models captured simulated large homozygous effects with and without heterozygous effects. When no heterozygous effect was present (i.e., a traditional recessive model), an additive model did not capture rare Mendelian homozygous effects well in 1,000 simulations (median p-value 0.20 , genome-wide significance in 1/1000) while the recessive model did (median p-value $1 \times 10 \mathrm{e}-10$, genomewide significance in 912/1000 simulations). However, with increasing heterozygous effect sizes, the additive model's significance increased (see Supplementary Figure S4). It is therefore likely that heterozygous effects contributed to our global phenotype association of variants with recessive inheritance. To confirm empirically, we repeated our global disease association after excluding 76 variants with homozygotes from our dataset of 311 variants. While reducing the number of variants expectedly reduced the signal, we still observed significantly (empirical p-value $<0.001$ ) more disease associations with $p$-values $<10^{-4}$ than expectation. Overall, these results suggest multiple variants with known recessive inheritance have modest heterozygous effects. We further explored heterozygous effects in five P/LP variants in genes SERPINA1, XPA, NPHS1, EYS and CLRN1 that were associated with diseases with $\mathrm{p}<5 \times 10^{-8}$. Variants in SERPINA1, XPA and NPHS1 still had nominally significant effects after removing homozygotes from the GWAS. The GWAS' phenotypes were similar to the known recessively inherited OMIM phenotypes (SERPINA1 -Emphysema, XPA - skin cancer, NPHS1 - nephrotic syndrome). For variants in genes SERPINA1 and NPHS1 we found that heterozygotes also had significantly earlier disease onset than wildtypes (p-value 0.05 , corrected for 5 tests, method: Wilcoxon-rank test). Expecting more heterozygous effects with increasing sample sizes, we found a heterozygous effect of a truncating variant in GJB2 on hearing loss (Figure $3 C, p=0.02$, beta $=0.11$ ) with a larger FinnGen dataset $(R 7, n=309,154)$. We would like to highlight the gene XPA where homozygous loss-of-function variants are known to cause the disease Xeroderma pigmentosum, a condition with extreme vulnerability to UV radiation and childhood-onset skin cancer ${ }^{33}$. (No homozygotes were present in FinnGen.) We found that a known pathogenic LLoF $^{29}$ (probable loss-of-function) variant in this gene was associated with adult-onset non-melanoma skin cancer in FinnGen in heterozygous state $\left(p=8 \times 10^{-11}\right.$, beta $=1.24$ corresponding to odds ratio=3.5). For longitudinal data see Figure $3 \mathrm{~B}$. The same variant was previously associated with basal cell carcinoma in Japan with similar effect size ${ }^{34}$ (odds ratio $=3.08$, $p$-value $=0.0097$ ). Thus, here we unequivocally confirm heterozygosity for XPA LoF is a significant risk factor for cancer and, given the high frequency of this variant in Finns, could be valuable personal risk information. 
Widening our search to variants that were described as P/LP by at least one but not all submitters in ClinVar and variants in genes with recessive AND dominant

227 inheritance in OMIM, we highlight a variant in SCN5A associated with severe arrhythmia 228 disorders like sick sinus syndrome ${ }^{35}$ in homozygous (or compound heterozygous) state, 229 which is confirmed in our dataset (Fisher's Exact test, p-value: 9x10-4,OR: 48 (95\%-Cl 6$230319)$ ). The same SCN5A variant however protected from arrhythmia diseases in FinnGen

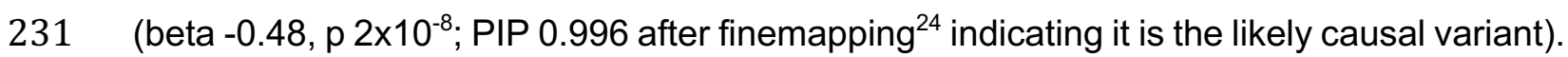

232 This included atrial fibrillation (beta -0.62 , p $7 \times 10^{-7}$ ) and could be replicated in the UKBB ${ }^{36}$ 233 (beta $-0.39, \mathrm{p} 0.04$ ).

234 In summary, we find global as well as individual association signals of variants previously 235 described to cause disease with recessive inheritance, semidominant inheritance in tumor 236 suppressor genes and disease associations of variants labelled B/LB. Our data thus 237 indicate the need for a nomenclature that better appreciates more complex inheritance of 238 Mendelian diseases. We outline a suggestion in Figure 2.

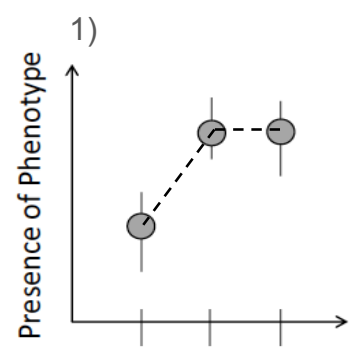

2)

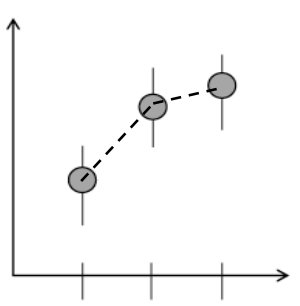

$A A \quad A B \quad B B$
3)

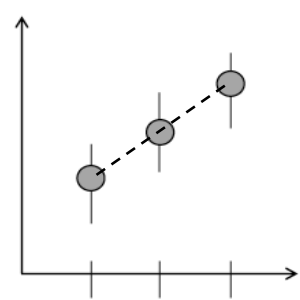

$A A \quad A B \quad B B$
4)

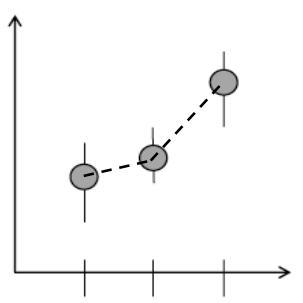

$A A \quad A B \quad B B$
5)

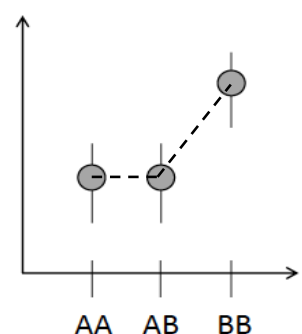

\begin{tabular}{l|l|l|l|l|l}
$\begin{array}{l}\text { Inheritance } \\
\text { (rare } \\
\text { disease) }\end{array}$ & Dominant & $\begin{array}{l}\text { Dominant, } \\
\text { with reduced penetrance } \\
\text { and/or variable expressivity } \\
\text { in heterozygotes }\end{array}$ & Semidominant & $\begin{array}{l}\text { Recessive, } \\
\text { with rare expressing } \\
\text { heterozygotes }\end{array}$ & Recessive \\
\hline $\begin{array}{l}\text { Genotype } \\
\text { dosage } \\
\text { effect }\end{array}$ & $\begin{array}{l}\text { Biallelic effect } \\
\text { equals } \\
\text { heterozygous effect } \\
\text { (nonlinear) }\end{array}$ & $\begin{array}{l}\text { Biallelic effect } \\
\text { marginally larger than } \\
\text { heterozygous effect } \\
\text { (nonlinear) }\end{array}$ & $\begin{array}{l}\text { Biallelic effect } \\
\text { double of } \\
\text { heterozygous effect } \\
\text { (additive, linear) }\end{array}$ & $\begin{array}{l}\text { exceeding } \\
\text { heterozygous effect } \\
\text { nonlinearly }\end{array}$ & $\begin{array}{l}\text { Biallelic effect, } \\
\text { no } \\
\text { heterozygous effect } \\
\text { (nonlinear) }\end{array}$ \\
\hline Remark & & & Standard GWAS model & &
\end{tabular}

Figure 2. Schema of different effect sizes of heterozygous (mono-allelic) vs. bi-allelic variant states. $A$ is the wildtype, $B$ is the mutant allele. We distinguish five main scenarios that are associated with different inheritance modes used in rare disease genetics (table's

252 first row). In rare disease genetics, the phenotypes associated with the mono- and bi-allelic

253 state in scenarios 2), 3) and 4) are usually viewed as distinct disease entities, with the 254 mono-allelic phenotype regarded as dominantly inherited and the usually more severe bi255 allelic phenotype regarded as recessively inherited. In the schema we don't show 256 overdominant/underdominant inheritance (rare outside the HLA region) and focus on 257 autosomal inheritance. 
A

Strata - wildtype - heterozygous - homozygous ALT

260

B

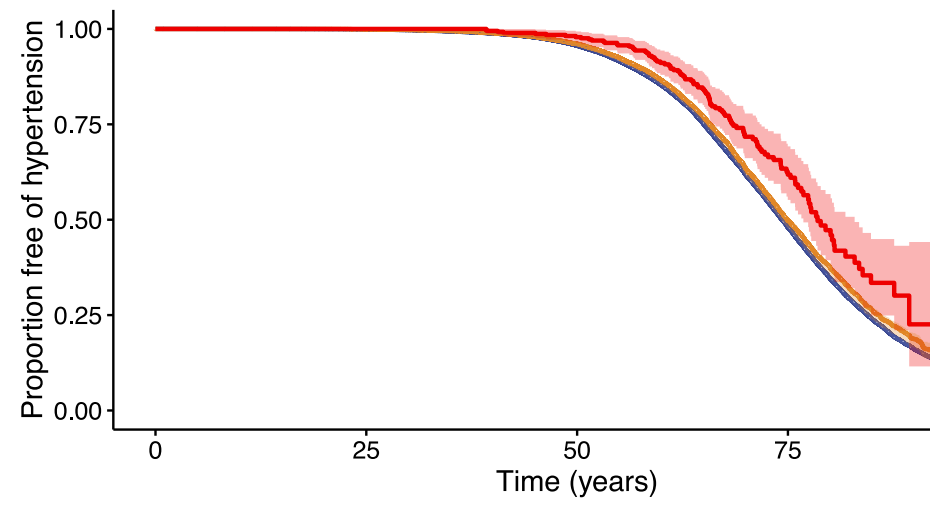

Strata - wildtype - heterozygous

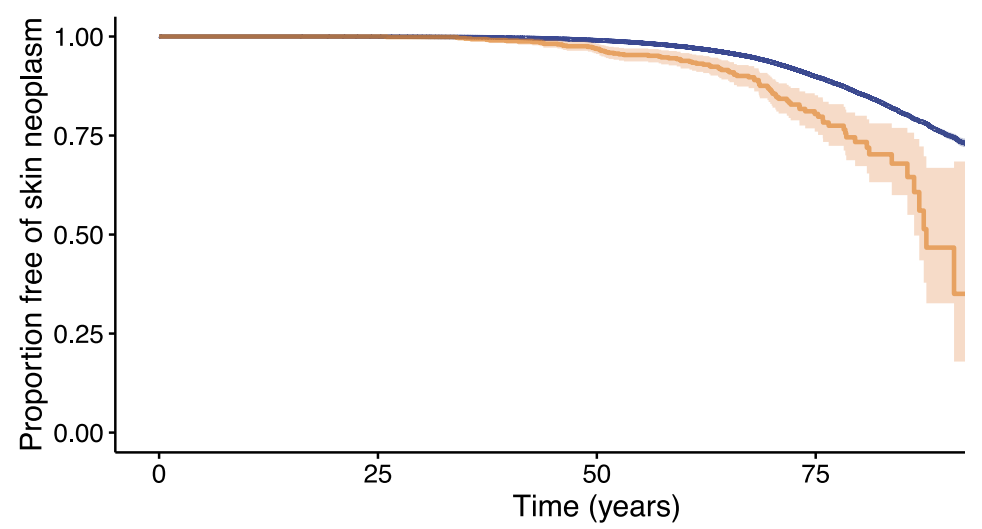

C

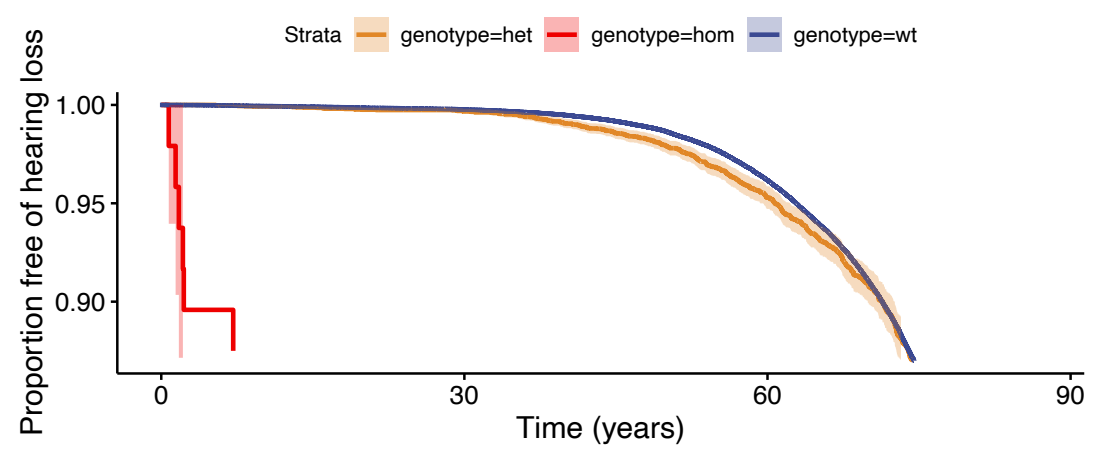

284

Figure 3. Age at first diagnosis of coding variant versus wildtypes (survival plots). A) $\mathrm{B} / \mathrm{LB}$ missense variant in the gene $D B H$ protects from hypertension. ( $D B H$ is associated 287 with the recessively inherited disease dopamine beta-hydroxylase deficiency which is 288 characterized by severe hypotension ${ }^{32}$ ), B) Known pathogenic variant in XPA is associated 289 with skin cancer. In homozygous state that variant causes Xeroderma pigmentosum with 290 childhood-onset skin cancer ${ }^{33}$. C) Known pathogenic variant (known recessive inheritance) 291 in GJB2 associated with hearing loss also in heterozygous state. The y-axis is cut at 0.9 292 for clarity. 


\begin{tabular}{|c|c|c|c|c|c|c|c|c|}
\hline Gene & GRCh38 & rsid & AF & OMIM phenotype of gene & $\begin{array}{l}\text { GWAS phenotype in } \\
\text { FinnGen }\end{array}$ & beta & p-value & PIP \\
\hline ARMS2 & $\begin{array}{l}10: 122454 \\
932: G: T\end{array}$ & $\begin{array}{l}\text { rs10490 } \\
924\end{array}$ & 0.24 & $\begin{array}{l}\text { Age-related macular } \\
\text { degeneration (association) }\end{array}$ & $\begin{array}{l}\text { Age-related mac. degen. } \\
\text { Other retinal disorders } \\
\text { Diabetic retinopathy }\end{array}$ & $\begin{array}{l}1.06 \\
0.27 \\
0.16\end{array}$ & $\begin{array}{l}1.90 \mathrm{E}-104 \\
1.78 \mathrm{E}-46 \\
3.92 \mathrm{E}-22\end{array}$ & $\begin{array}{l}0.08 \\
0.08 \\
0.08\end{array}$ \\
\hline ATM & $\begin{array}{l}11: 108272 \\
729: C: G\end{array}$ & $\begin{array}{l}\text { rs } 18000 \\
57\end{array}$ & 0.01 & $\begin{array}{l}\text { Ataxia-telangiectasia AR; } \\
\text { Breast cancer susceptibility }\end{array}$ & $\begin{array}{l}\text { Malignant neoplasm } \\
\text { Leiomyoma of uterus }\end{array}$ & $\begin{array}{l}0.28 \\
0.45\end{array}$ & $\begin{array}{l}2.58 \mathrm{E}-08 \\
1.13 \mathrm{E}-09\end{array}$ & $\begin{array}{l}0.4 \\
0.22\end{array}$ \\
\hline INS & $\begin{array}{l}11: 215983 \\
0: T: G\end{array}$ & $\begin{array}{l}\text { rs38427 } \\
53\end{array}$ & 0.21 & $\begin{array}{l}\text { Diabetes m. (neonatal, AR, } \\
A D, M O D Y \text { ) }\end{array}$ & $\begin{array}{l}\text { Diabetic maculopathy } \\
\text { Diabetic retinopathy }\end{array}$ & $\begin{array}{l}0.32 \\
0.32\end{array}$ & $\begin{array}{l}1.04 \mathrm{E}-10 \\
5.55 \mathrm{E}-19\end{array}$ & $\begin{array}{l}0.44 \\
0.51\end{array}$ \\
\hline HNF1A & $\begin{array}{l}\text { 12:120978 } \\
847: A: C\end{array}$ & $\begin{array}{l}\text { rs11692 } \\
88\end{array}$ & 0.37 & Diabetes m. (AR, AD MODY) & Statin medication & 0.08 & $1.55 \mathrm{E}-14$ & 0.86 \\
\hline KRT5 & $\begin{array}{l}12: 525198 \\
84: C: T\end{array}$ & $\begin{array}{l}\text { rs11170 } \\
164\end{array}$ & 0.1 & $\begin{array}{l}\text { Epidermolysis bullosa simplex, } \\
A R, A D\end{array}$ & $\begin{array}{l}\text { Malignant neoplasm of } \\
\text { skin }\end{array}$ & 0.21 & 1.34E-12 & 0.94 \\
\hline$R P L 3 L$ & $\begin{array}{l}\text { 16:194706 } \\
3: G: A\end{array}$ & $\begin{array}{l}\text { rs } 14797 \\
2626\end{array}$ & 0.01 & Cardiomyopathy, dilated, AR & $\begin{array}{l}\text { Atrial fibrillation and } \\
\text { flutter }\end{array}$ & 0.45 & $6.23 E-11$ & 0.49 \\
\hline TYK2 & $\begin{array}{l}\text { 19:103524 } \\
42: G: C\end{array}$ & $\begin{array}{l}\text { rs } 34536 \\
443\end{array}$ & 0.03 & Immunodeficiency, AR & $\begin{array}{l}\text { KELA Reimbursement } \\
\text { (Gastrointestinal) }\end{array}$ & -0.3 & 6.09E-09 & 0.7 \\
\hline $\mathrm{CFH}$ & $\begin{array}{l}\text { 1:1966901 } \\
07: C: T\end{array}$ & $\begin{array}{l}\text { rs10611 } \\
70\end{array}$ & 0.44 & $\begin{array}{l}\text { Complement factor } \mathrm{H} \\
\text { deficiency, AR, AD; } \\
\text { Macular deg. (association) }\end{array}$ & $\begin{array}{l}\text { Disorders of choroid and } \\
\text { retina } \\
\text { Diabetic retinopathy }\end{array}$ & $\begin{array}{l}-0.11 \\
-0.09\end{array}$ & $\begin{array}{l}5.18 \mathrm{E}-16 \\
2.05 \mathrm{E}-10\end{array}$ & $\begin{array}{l}0.02 \\
0.02\end{array}$ \\
\hline PCSK9 & $\begin{array}{l}1: 5503997 \\
4: G: T\end{array}$ & $\begin{array}{l}\text { rs11591 } \\
147\end{array}$ & 0.04 & $\begin{array}{l}\text { Hypercholesterolemia, familial, } \\
\text { AD }\end{array}$ & $\begin{array}{l}\text { Hypercholesterolaemia } \\
\text { Coronary } \\
\text { atherosclerosis } \\
\text { Myocardial infarction } \\
\text { Coronary revascular. } \\
\text { Statin medication }\end{array}$ & $\begin{array}{l}-0.43 \\
-0.22 \\
-0.32 \\
-0.36 \\
-0.61\end{array}$ & $\begin{array}{l}1.33 \mathrm{E}-16 \\
4.59 \mathrm{E}-09 \\
1.48 \mathrm{E}-11 \\
1.10 \mathrm{E}-12 \\
5.26 \mathrm{E}-119\end{array}$ & $\begin{array}{l}1 \\
0.41 \\
0.94 \\
0.93 \\
1\end{array}$ \\
\hline HNF4A & $\begin{array}{l}20: 444137 \\
24: C: T\end{array}$ & $\begin{array}{l}\text { rs18009 } \\
61\end{array}$ & 0.05 & MODY, type I, AD & $\begin{array}{l}\text { Cholelithiasis } \\
\text { Intrahepatic Cholestasis } \\
\text { of Pregnancy }\end{array}$ & $\begin{array}{l}0.38 \\
0.82\end{array}$ & $\begin{array}{l}1.27 E-30 \\
2.43 E-09\end{array}$ & $\begin{array}{l}1 \\
1\end{array}$ \\
\hline AIRE & $\begin{array}{l}21: 442944 \\
11: C: T\end{array}$ & $\begin{array}{l}\text { rs } 74203 \\
920\end{array}$ & 0.04 & $\begin{array}{l}\text { Autoimm. polyendocrinopathy } \\
\text { syndrome AR, AD }\end{array}$ & $\begin{array}{l}\text { Diabetic maculopathy } \\
\text { Type } 1 \text { diabetes } \\
\text { Diabetic retinopathy }\end{array}$ & $\begin{array}{l}0.64 \\
0.38 \\
0.48\end{array}$ & $\begin{array}{l}2.39 \mathrm{E}-09 \\
2.19 \mathrm{E}-10 \\
9.48 \mathrm{E}-11\end{array}$ & $\begin{array}{l}0.99 \\
0.96 \\
1\end{array}$ \\
\hline IFIH1 & $\begin{array}{l}2: 1622675 \\
41: C: T\end{array}$ & $\begin{array}{l}\text { rs19907 } \\
60\end{array}$ & 0.42 & $\begin{array}{l}\text { Aicardi-Goutieres AD; } \\
\text { Singleton-Merten, AD }\end{array}$ & $\begin{array}{l}\text { Hypothyroidism } \\
\text { Disorders of the thyroid } \\
\text { gland }\end{array}$ & $\begin{array}{l}0.08 \\
0.07\end{array}$ & $\begin{array}{l}8.88 \mathrm{E}-11 \\
5.43 \mathrm{E}-09\end{array}$ & $\begin{array}{l}0.62 \\
0.51\end{array}$ \\
\hline$A P O B$ & $\begin{array}{l}2: 2104102 \\
8: G: A\end{array}$ & $\begin{array}{l}\text { rs13671 } \\
17\end{array}$ & 0.28 & $\begin{array}{l}\text { Hypercholesterolemia AD } \\
\text { Hypobetalipoproteinemia, AR }\end{array}$ & $\begin{array}{l}\text { Disorders of lipoprotein } \\
\text { metabolism }\end{array}$ & 0.11 & $6.64 \mathrm{E}-12$ & 0.51 \\
\hline ZAP70 & $\begin{array}{l}\text { 2:9772515 } \\
\text { 3:C:T }\end{array}$ & $\begin{array}{l}\text { rs } 14595 \\
5907\end{array}$ & 0.02 & $\begin{array}{l}\text { Autoimmune disease, infantile, } \\
\text { AR }\end{array}$ & Hypothyroidism & 0.24 & 1.83E-08 & 0.43 \\
\hline$\angle R B A$ & $\begin{array}{l}4: 1502779 \\
28: G: A\end{array}$ & $\begin{array}{l}\text { rs22908 } \\
46\end{array}$ & 0.22 & $\begin{array}{l}\text { Immunodeficiency, with } \\
\text { autoimmunity, AR }\end{array}$ & $\begin{array}{l}\text { Cholelithiasis } \\
\text { Disorders of biliary tract } \\
\text { and pancreas }\end{array}$ & $\begin{array}{l}0.12 \\
0.1\end{array}$ & $\begin{array}{l}1.31 \mathrm{E}-13 \\
2.19 \mathrm{E}-12\end{array}$ & $\begin{array}{l}0.52 \\
0.7\end{array}$ \\
\hline$D B H$ & $\begin{array}{l}\text { 9:1336366 } \\
06: C: T\end{array}$ & $\begin{array}{l}\text { rs } 77273 \\
740\end{array}$ & 0.05 & $\begin{array}{l}\text { Orthostatic hypotension due to } \\
\text { DBH deficiency, AR }\end{array}$ & Hypertensive diseases & -0.16 & $6.16 \mathrm{E}-13$ & 0.99 \\
\hline
\end{tabular}

294 Table 1. B/LB ClinVar variants that are the most likely causative (top PIP) variant in

295 one GWAS locus. Genomic locations are given as rsids and GRCh38 coordinates

296 (chromosome, position in bp, reference and alternate allele, separated by ":"). PIP,

297 posterior inclusion probability (statistical finemapping ${ }^{24}$ ); $A F$, allele frequency in FinnGen;

298 AD, autosomal dominant; AR autosomal recessive; KELA, Finnish Social Insurance

299 Institution. All variants listed in Table 1 are missense variants. 
20 homozygous disease associations, of which 13 not found with additive models

302 To find potentially novel homozygous disease associations, we performed a pheWAS with 303 additive and recessive models of 82,647 coding variants in FinnGen (2,634 pLoF, 76,884 304 missense, 3,129 others, INFO > 0.8) on 2,444 disease endpoints in 176,899 Finns using $305 \mathrm{SAiGE}^{27}$. After excluding the HLA region (chr6:25Mb-35Mb), we found 1780 additive 306 associations with $p$-value $<5 \times 10^{-8}$ for 443 coding variants in 303 genes. (For further 307 information on these results see the FinnGen flagship paper, browse publicly released 308 summary statistics at http://r4.finngen.fi/ or download at 309 https://www.finngen.fi/en/access_results.) We then searched for homozygous effects 310 across all coding variants comparing homozygous with heterozygous+wildtype alleles. We 311 identified 124 associations - involving 39 unique variants - with a genome-wide significant $312 p$-value in the recessive GWAS model that was two orders of magnitude lower than the 313 additive GWAS $p$-value (Figure 4A). Considering adjacent variants with $r^{2}>0.25$ associated 314 with the same (parent) trait as one locus, these associations corresponded to 31 unique 315 loci (Figure 4B, Supplementary Table S2, Supplementary Figure S5). Genes GJB2 and 316 EYS each harbored two P/LP variants that were each significantly associated in the 317 recessive model with hearing loss (variants in GJB2) or retinal dystrophy (variants in EYS). 318 So we assume that here multiple disease-causing variants are causally associated at the 319 respective loci. We confirmed this with comp-het effects for the P/LP variants in GJB2 (see 320 Figure 5C) and additionally found comp-het effects in CERKL (see Supplementary Note 321 4). We next searched how many of the homozygous associations we identified were 322 already known. 13 out of the 31 lead variants were in known OMIM genes (Figure 4B) of 323 which all 13/13 genes were previously described with recessive disease inheritance in 324 OMIM with the phenotype matching the known OMIM phenotype in 12/13 genes. 9/13 of 325 variants in OMIM genes were previously reported disease-causing in $\mathrm{ClinVar}^{28}$ (6 [likely] 326 P/LP, 3 conflicting). As expected, known Mendelian disease variants had large effect sizes 327 with beta $>4$ (corresponds to an odds ratio of 55). Of the 22 variants that were not 328 previously reported disease-causing in ClinVar, 13/22 had Mendelian effect sizes with 329 homozygous beta $>4$ and were rare with MAF $<0.05$. They included novel disease genes 330 such as CASP7 and EBAG9 for which longitudinal disease onset data are shown in Figure 3315 and discussed in Supplementary Note 5 . In addition, we find that homozygotes of the 332 EBAG9 variant have fewer children and a later age at first child than wildtypes 333 (Supplementary Figure S6). The association of C10orf90 with hearing loss was published 334 during the revision of this manuscript ${ }^{37}$. We thus provide a well-powered replication and 335 additional information on disease onset (Figure 5). Our six most common variants with 336 homozygous effects (MAF > 0.1) overlapped known associations in the GWAS catalog 337 (Supplementary Table S3, variants in LD with $r^{2}>0.1^{38}$ ). For $4 / 6$ of those variants, their 
phenotype in FinnGen matched the associated known GWAS hit. In addition, the variant in gene TMEM214 that we found to be protective against pain (including limb, neck, head pain) in FinnGen in a homozygous state was in LD $\left(r^{2} 0.78\right)$ with a GWAS hit for CRP level. It seems biologically plausible that CRP as a standard biomarker for systemic inflammation may be associated with unspecified pain.

We next sought to validate any homozygous associations in the UK biobank ${ }^{36}$ and 344 in the FinnGen datafreeze R6. Owing to the Finnish population's isolation ${ }^{10}$ (Figure 1, 345 introduction), most of our variants with homozygous effects were Finnish-enriched with the 346 exception of variants in genes GJB2, SERPINA1 and C15orf40 being $>2$ fold higher in 347 Finnish than non-Finnish-Swedish-Estonian Europeans in gnomAD. In total, 13/31 variants 348 had $>=5$ homozygotes and partially matching phenotypes in UKBB suitable for validation. $3498 / 13$ of those had significant homozygous associations ( $p$-value $<0.05$ ) to related 350 phenotypes in the UKBB (Supplementary Figure S7, Supplementary Table S4). For 3 variants with $<5$ homozygotes in UKBB, we found homozygous associations ( $p$-value < $0.05)$ in other coding variants in the UKBB. In addition, 8 variants had additive associations ( $p$-value < 0.05) in the UKBB (Supplementary Table S5). 18/31 variants stayed genome-

354 wide significant in FinnGen datafreeze R6 resulting in a total of 20 homozygous 355 associations validated in FinnGen R6 and/or UKBB (Table 2). These included 4/20 novel 356 associations. Homozygous associations that could not be validated are listed in 357 Supplementary Table S2. The majority of homozygotes also had substantially earlier 358 disease onset than wildtypes when affected with the same diseases, which we interpret as additional evidence for genetic variants' effect on disease (Supplementary Figure S8).

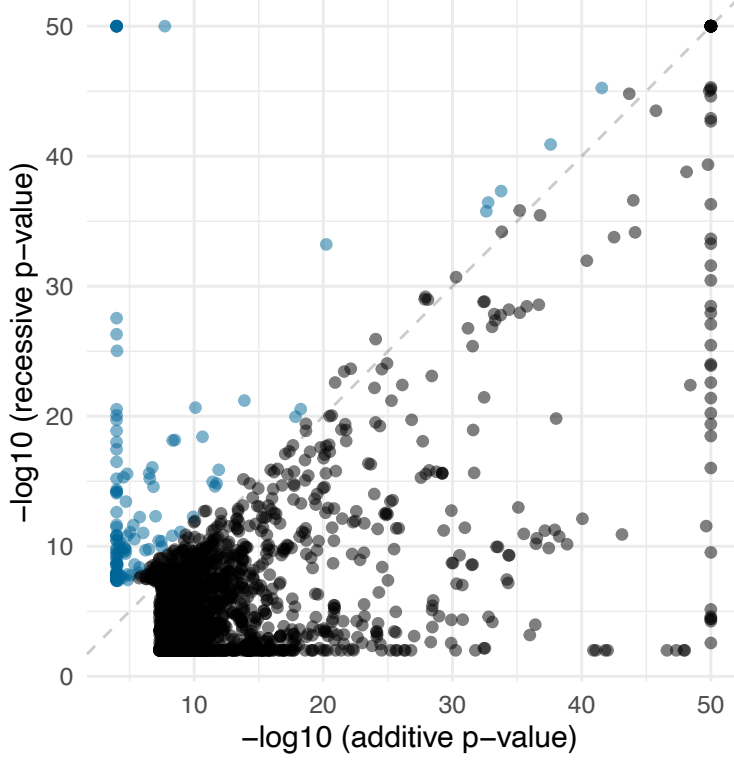




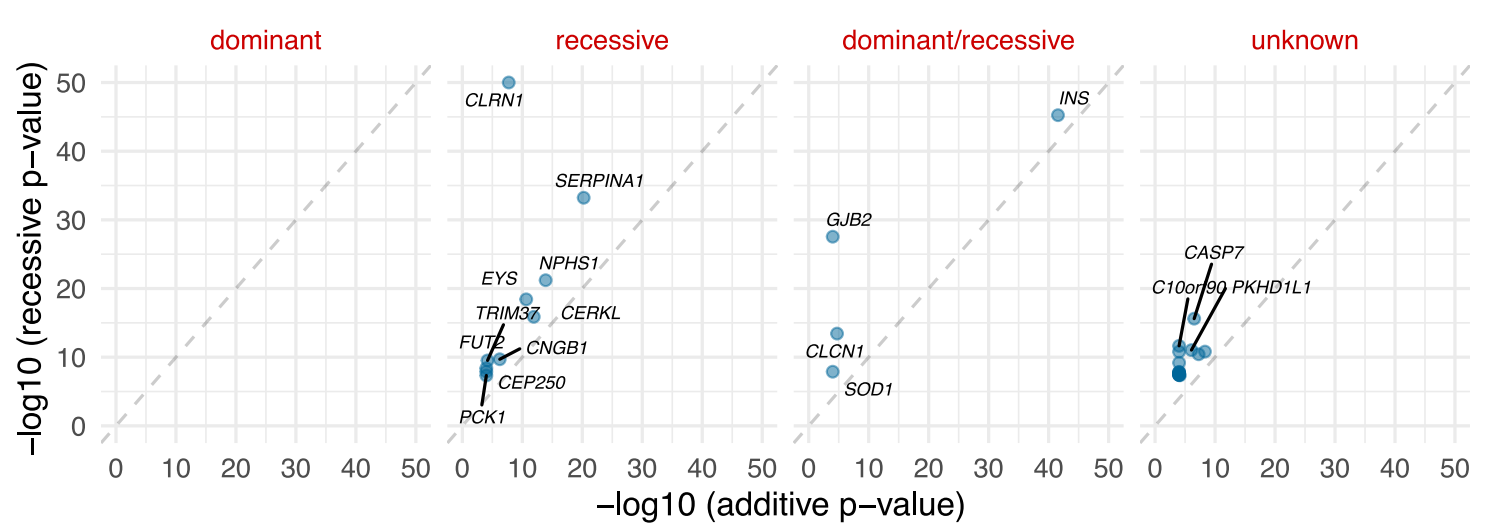

Figure 4. P-values of the additive vs. recessive GWAS model of all genome-wide significant variant-phenotype associations. Associations with recessive model's pvalue two orders of magnitude lower than additive model's p-value are shaded in blue (=category "recessive"), all other associations are shaded in black. A) All associations. B) Recessive associations, independent loci, broken down by listed inheritance modes of the respective Mendelian genes in OMIM. For clarity, -log10 p-values are capped at 50.

We next investigated if any of the variants more significant in the recessive than additive model also had subtle heterozygous effects on the same disease endpoints by excluding homozygotes from GWAS. Four variants had nominally significant heterozygous effects. These included variants in SERPINA1 (phenotype: Emphysema, p-value: 0.037, beta: 0.5), NPHS1 (phenotype: nephrotic syndrome, p-value: 0.0046, beta: 1.2), CERKL (phenotype: retinal dystrophy, p-value: 0. 0035, beta: 2.7 ) and CASP7 (phenotype: adultonset cataract, p-value: 0.0035 , beta: 0.25 ). However, when excluding individuals with comp-het variants from the analysis, the association for CERKL dropped to $p=0.14$, beta 1.4 indicating the heterozygous effect was driven by comp-hets while the association in NPHS1 stayed significant ( $\mathrm{p}$-value 0.0004 , beta 1.3 ). We thus classify inheritance of variants in NPHS1, CASP7 and SERPINA1 as recessive, with rare expressing heterozygotes (schema: Figure 2).

$13 / 20$ of our validated homozygous associations were not genome-wide significant in the additive GWAS model (Supplementary Table 2). In simulations, an additive model found a homozygous effect (10x higher disease probability in homozygotes, MAF 0.01 in $n=200,000$ ) in $1 / 1000$ simulations while the recessive model found it in $912 / 1000$ simulations (Supplementary Note 3). This illustrates how using a recessive GWAS model may uncover associations that might otherwise be missed (Supplementary Figure S4). 


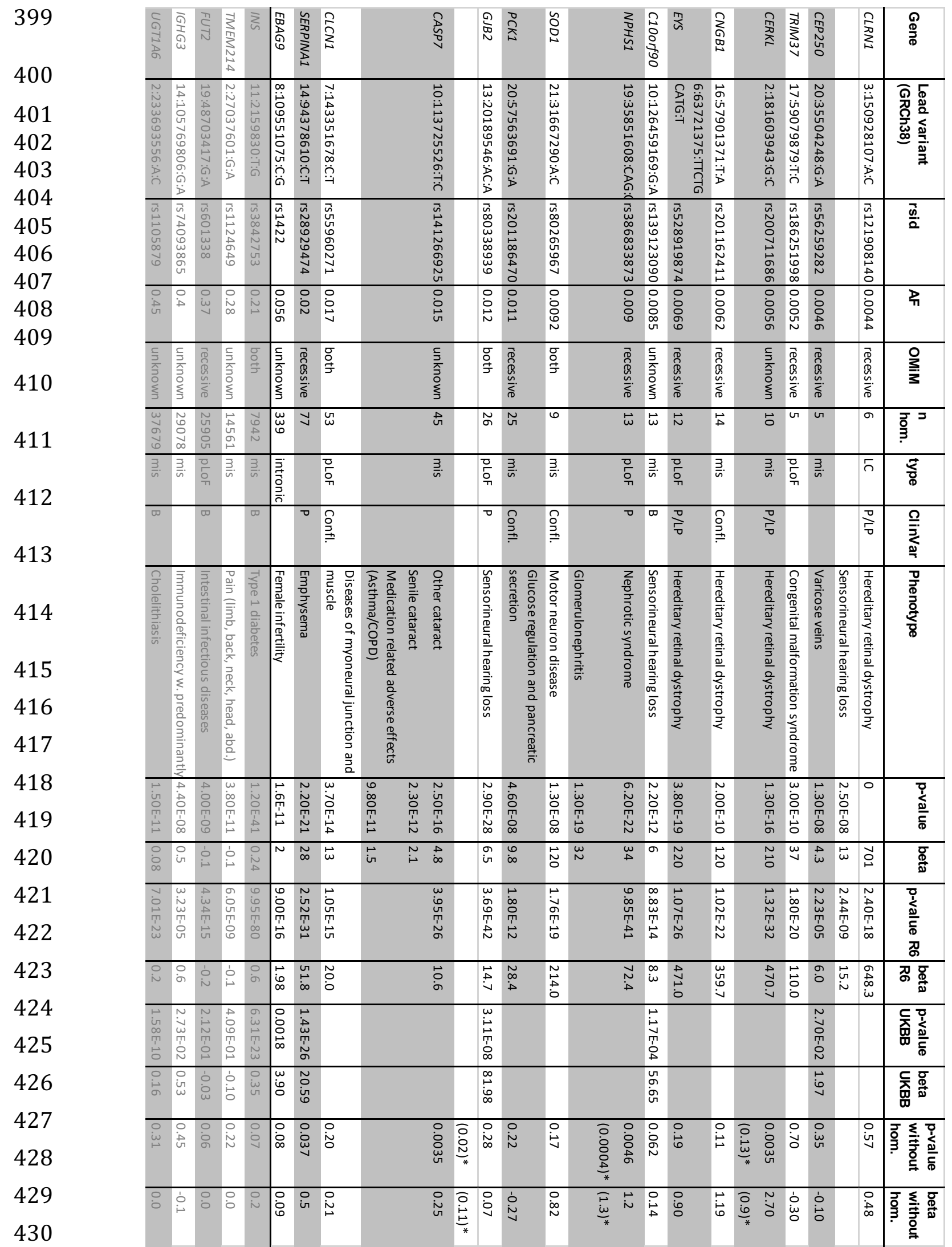


432 Table 2. 20 homozygous associations. mis, missense; pLoF, probable loss-of-function;

433 AF, allele frequency in FinnGen; hom, homozygotes in FinnGen; R6, FinnGen data release

$4346(n=234,553)$; UKBB, UK biobank $(n=420,531)$. Adjacent variants with $r^{2}>0.25$ associated

435 with the same (parent) trait are considered as one locus and only the most significant

436 variant shown per locus. Lead variants are given as rsids and GRCh38 coordinates

437 (chromosome, position in bp, reference and alternate allele, separated by ":"). The p-value

438 and beta in ( $)^{*}$ refers to the repetition of the heterozygous test in $R 6$ or $R 7$ data $(n=309,154$,

439 only GJB2) after exclusion of comp-het carriers. EBAG9 is the only noncoding variant

440 presented here, as we estimate it is a more likely causal variant than the originally identified

441 pLoF in PKHD1L1 (Supplementary Note 5). Variants at the bottom shaded in grey overlap

442 known GWAS hits loci that are listed in Supplementary Table S3.

443

444

A)

Strata - genotype=het - genotype=hom - genotype=wt

445

446

447

448

449

450

451

452

453

B)

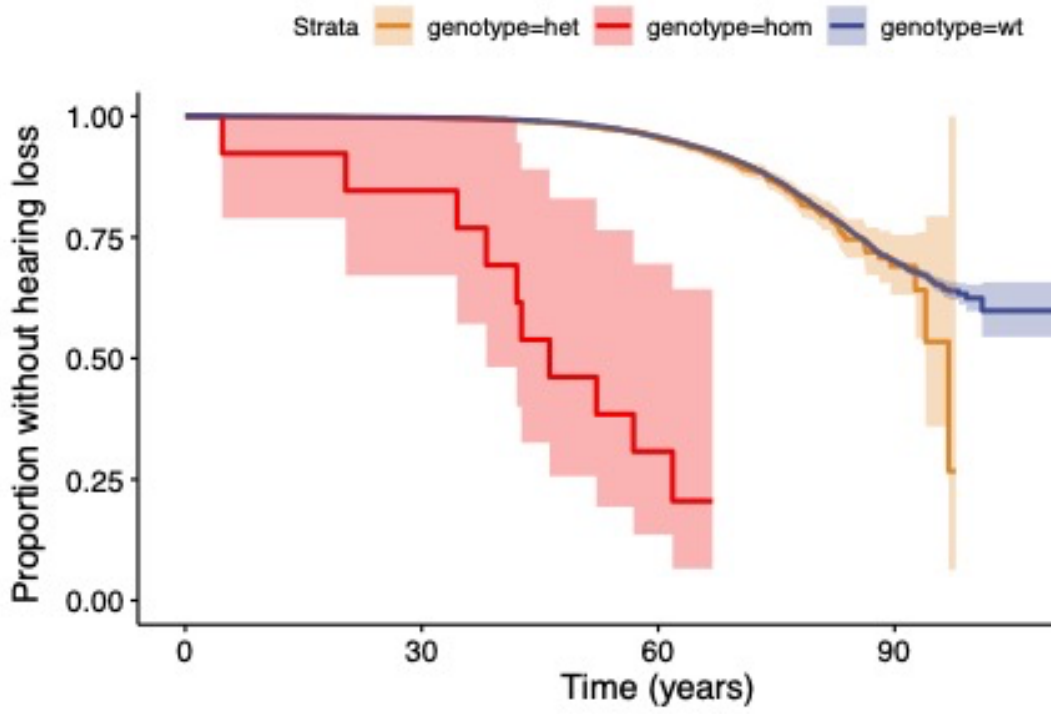

454

455

456

457

458

459

460

461

462

463

464

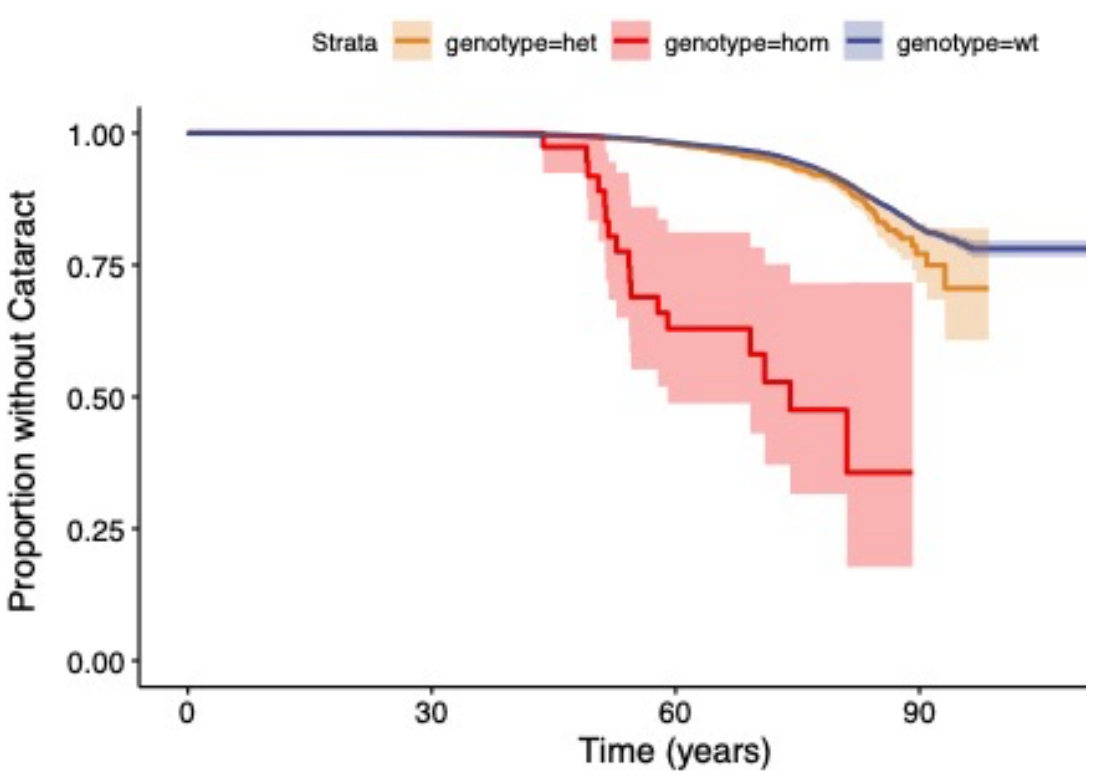

465 
C)

468

genotype=het

genotype=hom

genotype=wt

469

470

471

472

473

474

475

476

477

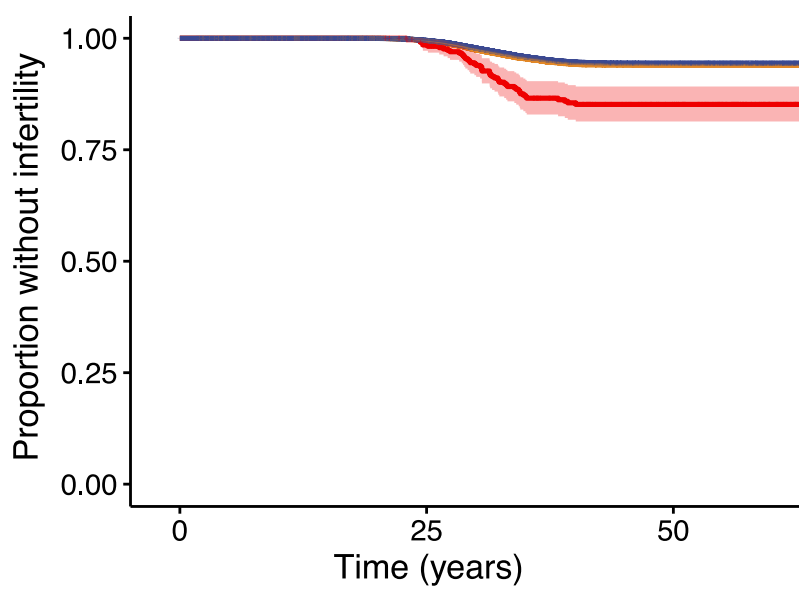

478

479 Figure 5. Age at first disease diagnosis of variant versus wildtypes (survival plots).

480 Wt, wildtype; het, heterozygous; hom, homozygous. A) Missense variant in C10orf90

481 associated with hearing loss, only recently described ${ }^{37}, n=176,899$. B) Missense variant in

482 CASP7 associated with cataract, not previously described, $n=176,899$. C) Intronic variant

483 in EBAG associated with female infertility, not previously described, $n=110,361$ females.

484 


\section{Discussion}

A subset of disease-causing variants are enriched to unusually high frequencies in populations with a history of recent bottlenecks such as Finland. This applies particularly to homozygous variants causing disease with recessive inheritance. The FinnGen cohort, given its size and broad medical phenotypes over the lifespan, is therefore well-powered to study both the effects of Mendelian mutations with known recessive inheritance in heterozygotes and to discover novel alleles with an outsized effect in homozygotes.

We first studied effects of variants previously reported in human disease in $\mathrm{ClinVar}^{28}$ in $>170 \mathrm{k}$ participants of FinnGen. Here, we found that multiple variants previously described as B/LB had effects on disease phenotypes which is noteworthy as a reminder that variants labeled as benign, even if they do not cause monogenic disease, should not be presumed to be neutral ${ }^{31}$ or without phenotypic consequence (see Supplementary Note 2). We then found genome-wide significant effects of 26 known disease-causing ClinVar variants including semidominant effects in tumor suppressor genes CHEK2, JAK2, OCA2 and MC1R that were in part previously described ${ }^{39-42}$. We also discovered that a heterozygous truncating variant in the tumor suppressor and DNArepair gene XPA increased susceptibility to adult-onset skin cancer with an effect far stronger than usually found by GWAS. This association was already shown with nominal significance $(p=0.01)$ in a previous study ${ }^{34}$. Thus, we provide the first definitive evidence of this link in heterozygotes. Homozygous loss-of-function variants in the gene XPA are known to cause the disease Xeroderma pigmentosum (XP), a condition with extreme vulnerability to UV radiation and childhood-onset skin cancer ${ }^{33}$. Given the phenotypic relatedness of skin cancer and XP and the semidominant effects of variants in other tumor suppressor genes, this highlights that our way of describing their respective inheritance with only dominant and recessive labels does not adequately capture disease biology. For distinct molecular effects, it makes however biologically sense to view their inheritance separately. SERPINA1 is an example where the $\mathrm{Pi}-\mathrm{Z}$ variant causes different disease entities with distinct molecular effects, which we can also observe in our data. The Pi-Z variant causes a molecular gain-of-function of Alpha1-Antitrypsin (A1AT) which is not

516 Cholelithiasis or Fibrosis. Improper secretion of A1AT however results in a deficiency at its

517 target tissue, the lung, a loss-of-function effect that would only manifest if A1AT level is 518 very low as in $\mathrm{Pi}-\mathrm{Z}$ homozygotes. The inheritance of $\mathrm{Pi}-\mathrm{Z}$ is dominant for the liver 519 phenotype, but recessive for the lung phenotype (with a small heterozygous effect). LDLR 520 associated with familial hypercholesterolemia $(\mathrm{FH})$ is another example for semidominant 521 inheritance where bi-allelic pathogenic variants result in more severe disease while single 
522 heterozygous pathogenic variants lead to a milder form of $\mathrm{FH}$. On OMIM, however, only

523 recessive and dominant inheritance modes are given for $\mathrm{FH}$ associated with $L D L R$. Terms

524 beyond recessive/dominant such as semidominant inheritance are rarely used in clinical

525 genetics ${ }^{43}$ even though recommended in ClinGen's gene curation SOP. While it has been

526 estimated that semidominant effects are much more common than true dominant effects ${ }^{44}$,

527 phenotypes of heterozygotes/homozygotes are frequently unknown. Semidominant

528 inheritance is more frequently described in animal and plant genetics ${ }^{45}$, where mono- and

529 bi-allelic effects can be more easily systematically studied. In addition to a more accurate

530 description of biology, it could benefit clinical variant interpretation if evidence for

531 pathogenicity was aggregated across bi-allelic and mono-allelic observations and not

532 viewed as separate disease entities.

533 Adding to an increased complexity of Mendelian variants' inheritance, we also detect a

534 global heterozygous effect on disease in variants known to cause disease with recessive

535 inheritance. This is in line with previous studies that found subtle disease and/or fitness

536 effects of heterozygous variants in genes described to cause disease with recessive

537 inheritance in mice ${ }^{46}$, drosophila ${ }^{47}$ but also in humans ${ }^{48}$. Individual examples include the

538 already-mentioned variant in XPA known to cause XP in a homozygous state ${ }^{33}$, that

539 increased risk of skin cancer while in a heterozygous state. We also find heterozygous

540 effects of variants with recessive inheritance in NPHS1 on kidney disease, which was

541 previously debated ${ }^{49}$, in SERPINA1 on emphysema ${ }^{50}$ and GJB2 on hearing loss,

542 previously hypothesized ${ }^{51}$. As expected for recessive inheritance, the variants had an

543 order of magnitude larger effect size and often earlier disease onset in the homozygous

544 than in heterozygous state thereby exceeding a linear additive model. With increasing

545 sample sizes, we identify more heterozygous effects indicating this could be a more

546 widespread phenomenon (e.g. in GJB2 we find the effect on hearing loss only with a larger

547 dataset $[n=309,154])$. Of course, it is possible that some heterozygous effects come from

548 low-frequency variants in comp-het state (as we observe for CERKL) that were not

549 captured by our genotyping array. However, a different age of disease onset of

550 heterozygotes than homozygotes suggests that scenario is unlikely. In addition, with

551 population specific exome sequencing and imputation we could account for the presence

552 of additional pathogenic variants at frequencies $>0.2 \%$. Another limitation of our approach

553 is the lack of more in-depth phenotypes such as serological or diagnostic tests. We thus

554 likely miss subtle physiological differences between heterozygotes and wildtypes. This

555 should, however, not affect our main conclusions. Long before these large-scale data

556 became available, small heterozygous effects were found in variants causing Mendelian

557 disease with recessive inheritance ${ }^{52}$, in some cases giving an advantage against certain

558 infectious diseases ${ }^{53,54,55}$. Similarly, we find that one variant in SCN5A, which was 
559 previously associated with severe arrhythmia disorders like sick sinus syndrome ${ }^{35}$ in a bi560 allelic state, protected from mild arrhythmia diseases including atrial fibrillation in a 561 heterozygous state in FinnGen. Previous experimental data found a mild loss-of-function 562 effect of this variant ${ }^{35,56}$. This is in line with some ECG times being longer in a few SCN5A 563 heterozygotes ${ }^{35}$ pointing to a potential slowing of electrical conduction in the heart outlining 564 a possible protective mechanism against cardiac arrhythmia such as atrial fibrillation. 565 Further studies are needed to elucidate the mechanism comprehensively.

566 We systematically investigated coding variants' homozygous effects genome-wide 567 with pheWAS at biobank-scale. We could validate 20 loci (4 of them novel) with large bi568 allelic effects without, or with only nominally significant, heterozygous effects. Known 569 associations (e.g. variants in FUT2 protecting from intestinal infection ${ }^{57}$ ) served as positive 570 controls. Novel associations include complex non-syndromic diseases with no/few 571 previously described large-effect variants like adult-onset cataract (new disease gene: $572 C A S P 7)$ and female infertility (new disease gene: EBAG9). Intuitively, these novel findings 573 appear in phenotypes (deafness, cataracts, infertility) for which Mendelian subtypes would 574 not obviously be clinically distinguished from other common presentations, though as in 575 past examples earlier age of onset in these cases is notably seen. Bi-allelic associations 576 of rare coding variants were found in other population biobanks ${ }^{9,58-60}$ though to our 577 knowledge, not investigated at that broad phenotype scale.

578 In summary, our biobank-scale additive and homozygous pheWAS of coding 579 variants demonstrate a benefit of homozygous scans in GWAS. We find known and novel 580 bi-allelic associations across a broad spectrum of phenotypes such as retinal dystrophy, 581 adult-onset cataract and female infertility that are missed by the standard additive GWAS 582 model. As a related point, we find evidence of complex inheritance patterns of multiple 583 Mendelian variants and highlight a more widespread presence of allelic series in clinically 584 relevant genes than previously appreciated. 


\section{Supplementary Material}

\section{Supplementary Figures}

589 Supplementary Figure S1. MAF of known disease-causing ClinVar variants in Finnish 590 disease heritage genes in FIN and NFE gnomAD cohorts.

Supplementary Figure S2A-AG (separate pdf). Longitudinal survival curves showing disease onset of homozygous, heterozygous and wildtypes of likely P/LP or conflicting variants.

Supplementary Figure S3. Global disease associations of variant categories.

598 Supplementary Figure S4. Simulations of rare recessive and additive effects with varying 599 sizes of heterozygous effects.

600

601 Supplementary Figure S5A-AE (separate pdf). Longitudinal survival curves showing 602 disease onset of homozygous, heterozygous and wildtypes of variants with homozygous 603 effects in FinnGen.

604

605 Supplementary Figure S6. EBAG is associated with female infertility.

606

607 Supplementary Figure S7. Replications of homozygous associations in the UK biobank.

608

609 Supplementary Figure S8. Age at first diagnosis of homozygous, heterozygous and wildtypes of variants with homozygous effects in FinnGen.

612 Supplementary Figure S9. Age at first disease diagnosis of variant carriers in GJB2.

\section{Supplementary Tables}

616 Supplementary Table 1. Most significant disease associations of all 15140 coding variants 617 annotated in ClinVar.

619 Supplementary Table 2. Summary statistics of coding variants with any homozygous

620 association with $p$-value $<10^{-7}$ (all available disease endpoints, associations with $p$-value $621<0.01)$. 
623 Supplementary Table 3. Variants with homozygous disease associations in FinnGen that

624 overlap known GWAS loci.

625

626 Supplementary Table 4. Variants with homozygous disease associations in FinnGen that 627 could be replicated in the UK biobank.

628

629 Supplementary Table 5. Variants with disease homozygous associations in FinnGen that 630 could be tested for additive association in the UK biobank.

631

\section{Supplementary Notes}

633

634 Supplementary Note 1. Known disease-causing variants in FinnGen.

635

636 Supplementary Note 2. B/LB variants in FinnGen.

637

638 Supplementary Note 3. Simulations of homozygous and additive effects.

639

640 Supplementary Note 4. Compound heterozygous effects in GJB2 and CERKL.

641

642 Supplementary Note 5. Novel homozygous disease associations in CASP7, C10orf90 and 643 EBAG9.

644

645

646 List of FinnGen contributors. 


\section{Acknowledgements}

649 We would like to thank all FinnGen participants for their contributions to research. Patients

650 and control subjects in FinnGen provided informed consent for biobank research, based

651 on the Finnish Biobank Act. Research cohorts collected prior the Finnish Biobank Act were

652 collected based on study-specific consents and later transferred to the Finnish biobanks

653 after approval by Fimea, the National Supervisory Authority for Welfare and Health. The

654 FinnGen project, data release 4, was funded by Business Finland and eleven industry

655 partners. Please find further information on funding and approvals in the Methods section

656 and the full list of members of the FinnGen consortium in the Supplementary Information.

657 We thank Leslie Biesecker, Alfred L. George and Jack Kosmicki for helpful comments.

\section{Author contributions}

659 H.O.H wrote the manuscript and generated the figures. H.O.H and J.K. performed 660 analyses. K.J.K., S.M.L., A.S.H, W.Z., M.K. and H.L.R. contributed data and tools. A.P. 661 and M.J.D. supervised the study. H.O.H and M.J.D. conceived the study. All authors listed 662 under FinnGen contributed to the generation of the primary data of the FinnGen data 663 release 4. All authors reviewed the manuscript.

\section{Competing interests}

665 A.P. is a member of the Pfizer Genetics Scientific Advisory Panel. M.J.D is a founder of 666 Maze Therapeutics. The remaining authors declare no competing interests. 
Data availability

671 All summary statistics described in this manuscript can be found in the Supplementary

672 Material. Additional summary statistics of additive and recessive GWAS of FinnGen

673 release 4 can be investigated in our web browser at http://r4.finngen.fi/ or downloaded at

674 https://www.finngen.fi/en/access results. A full list of FinnGen endpoints for release 4 is

675 available at https://www.finngen.fi/en/researchers/clinical-endpoints. We are currently

676 submitting variants with novel associations or variants that clarified conflicting or wrong 677 classifications to ClinVar.

678

679

Code availability

680 Please see https://finngen.gitbook.io/documentation/ for a detailed description of data 681 production and analysis including code used to run analyses. Please see 682 https://github.com/FINNGEN/ for further code repositories used to run analyses in 683 FinnGen. R code to reproduce figures are available upon request.

684

685 Ethics and Data Access Approvals.

686 The Coordinating Ethics Committee of the Hospital District of Helsinki and Uusimaa (HUS) 687 approved the FinnGen study protocol Nr HUS/990/2017. The FinnGen project is approved 688 by Finnish Institute for Health and Welfare (THL), approval number 689 THL/2031/6.02.00/2017, amendments THL/1101/5.05.00/2017, THL/341/6.02.00/2018, 690 THL/2222/6.02.00/2018, THL/283/6.02.00/2019), Digital and population data service 691 agency VRK43431/2017-3, VRK/6909/2018-3, the Social Insurance Institution (KELA) 692 KELA 58/522/2017, KELA 131/522/2018, KELA 70/522/2019 and Statistics Finland TK693 53-1041-17. The Biobank Access Decisions for FinnGen samples and data utilized in 694 FinnGen Data Freeze 4 include: THL Biobank BB2017_55, BB2017_111, BB2018_19, 695 BB_2018_34, BB_2018_67, BB2018_71, BB2019_7 Finnish Red Cross Blood Service 696 Biobank 7.12.2017, Helsinki Biobank HUS/359/2017, Auria Biobank AB17-5154, Biobank 697 Borealis of Northern Finland_2017_1013, Biobank of Eastern Finland 1186/2018, Finnish 698 Clinical Biobank Tampere MH0004, Central Finland Biobank 1-2017, and Terveystalo 699 Biobank STB 2018001. UK biobank data was accessed under protocol 31063.

701 Funding and Partners.

702 The FinnGen project is funded by two grants from Business Finland (HUS 4685/31/2016 703 and UH 4386/31/2016) and eleven industry partners (AbbVie Inc, AstraZeneca UK Ltd, 704 Biogen MA Inc, Celgene Corporation, Celgene International II Sàrl, Genentech Inc, Merck 
705 Sharp \& Dohme Corp, Pfizer Inc., GlaxoSmithKline, Sanofi, Maze Therapeutics Inc., 706 Janssen Biotech Inc). Following biobanks are acknowledged for delivering biobank 707 samples to FinnGen: Auria Biobank (www.auria.fi/biopankki), THL Biobank 708 (www.thl.fi/biobank), Helsinki Biobank (www.helsinginbiopankki.fi), Biobank Borealis of 709 Northern Finland (https://www.ppshp.fi/Tutkimus-ja-opetus/Biopankki/Pages/Biobank710 Borealis-briefly-in-English.aspx), Finnish Clinical Biobank Tampere (www.tays.fi/en711 US/Research_and_development/Finnish_Clinical_Biobank_Tampere), Biobank of

712 Eastern Finland (www.ita-suomenbiopankki.fi/en), Central Finland Biobank

713 (www.ksshp.fi/fi-Fl/Potilaalle/Biopankki), Finnish Red Cross Blood Service Biobank 714 (www.veripalvelu.fi/verenluovutus/biopankkitoiminta) and Terveystalo Biobank 715 (www.terveystalo.com/fi/Yritystietoa/Terveystalo-Biopankki/Biopankki/). All Finnish

716 Biobanks are members of BBMRI.fi infrastructure (www.bbmri.fi).

718 Coding variants in FinnGen, release 4

719 In summary, we investigated 82,647 coding variants in the FinnGen project, release 4 720 (2,634 pLoF, 76,884 missense, 3,129 others) in 176,899 Finns. In 110,361 individuals sex 721 was imputed as female. We filtered to variants with INFO $>0.8$ at a median MAF 0.004 722 (minimum MAF $3 \times 10^{-5}$ ). Circa half of the samples came from existing legacy collections 723 while the other half was from participants newly recruited to the FinnGen project. Samples 724 were genotyped on custom microarrays and rare variants imputed using a population 725 specific reference panel ${ }^{26}$. Please see the flagship paper or 726 https://finngen.gitbook.io/documentation/ for a detailed description of data production and 727 analysis. To calculate variant enrichment in Finns after a bottleneck event, we utilize as a 728 general European reference point exomes from European samples in gnomAD 2.1.1 729 excluding those from Finland, Sweden and Estonia (so-called non-Finnish-Swedish-

730 Estonian Europeans). Due to large-scale migrations from Finland to Sweden in the 20th 731 century, a substantial fraction of the genetic ancestry in Sweden is of recent Finnish origin, 732 and the linguistically (and geographically) close population of Estonia is likely to share 733 elements of the same ancestral founder effect.

735 Validation in FinnGen, release 6

736 We validated homozygous associations of the lead variants in FinnGen data release 6 737 consisting of genotype and EHR data from 234,553 Finns.

739 GWAS searching for additive, homozygous and heterozygous effects

740 We performed GWAS on 2,444 disease endpoints investigating the effects of 82,647 741 coding variants with an additive and recessive model using the method SAiGE ${ }^{27}$. 
742 Covariates in FinnGen were Age, Sex, genotyping batch and the first 10 Principal

743 Components of genotypes. To identify heterozygous effects we performed GWAS with an

744 additive model after excluding homozygotess. In the recessive GWAS model we analyzed

745 effects of homozygous alleles on disease phenotypes in comparison to

746 wildtype+heterozygous alleles. The docker container finngen/saige:0.39.1.fg with all

747 necessary software used to run SAiGE in additive or recessive mode can be found at the

748 docker container library hub.docker.com. We replicated our identified 31 genome-wide

749 significant homozygous associations in 420,531 individuals with European ancestry in the

750 UK biobank using the same recessive model in SAiGE. GWAS covariates in UKBB were

751 Age, Sex, Age $x$ Sex, $\mathrm{Age}^{2}, \mathrm{Age}^{2} \times$ Sex and the first 10 Principal Components of genotypes.

752 The genotype and phenotype files along with ancestry definitions, phenotype definitions

753 and SAiGE null models were taken from the PAN UKBB project and are further described

754 here https://pan.ukbb.broadinstitute.org.

755

756 Annotating variant effects from ClinVar

757 We annotated variants from release 03/25/2020 of ClinVar ${ }^{28}$. For any variant included in 758 the main tables, we rechecked current classifications in ClinVar and OMIM on 11/02/2021.

759 We grouped variants into categories according to their "ClinVar_ReviewStatus". Our main 760 categories were P/LP (= likely pathogenic or pathogenic, 311 variants), conflicting 761 evidence (=at least one submitter labeled a variant as P/LP but at least one other submitter 762 labeled it different from P/LP, 298 variants) and B/LB (= likely benign or benign, 10,948

763 variants). Other categories into which we grouped variants which we are not explicitly 764 discussing in the manuscript were 'association' (26 variants), 'drug response' (59 variants), 765 'not provided' (141 variants), 'protective' (14 variants), 'risk factor' (74 variants) and 'VUS' 766 (= variant of unknown significance, 3269 variants), see Supplementary Table 1.

768 Annotating inheritance mode from OMIM

769 We downloaded the Online Mendelian Inheritance in Man (OMIM) catalog of human 770 genetic diseases (www.omim.org) version 06/2019. From OMIM, we annotated genes 771 reported to have variants that cause disease with a recessive inheritance mode such as

772 NPHS1. Genes showing recessive as well as dominant inheritance modes with the same 773 or different phenotypes were labeled as "both OMIM genes" such as GJB2.

\section{Global phenotype associations of ClinVar variant categories}

776 We compared global disease phenotype associations of different ClinVar variant 777 categories (B/LB, P/LP or conflicting variants in genes with dominant or recessive 778 inheritance) with phenotype associations of random intergenic variants. For a given variant 
779 category, we counted how many variants had at least one significant GWAS hit $(2,444$ 780 phenotypes) below a given p-value threshold. We then compared those to the number of 781 top GWAS loci below the p-value threshold of 1000 random samples of intergenic variants. 782 We calculated with empirical p-values if any ClinVar variant categories had significantly 783 more disease associations than random intergenic variants below respective $p$-value 784 thresholds. Minor allele frequency (MAF) influences the power to identify significant 785 associations. Therefore we adjusted for MAF by sampling intergenic variants in 15 equal 786 sized bins that corresponded to the MAF of the variants under investigation. To account 787 for linkage disequilibrium we sampled intergenic variants from the same $3 \mathrm{Mb}$ windows as 788 variants in the respective gene set.

790 Age at first diagnosis

791 We compared age at first diagnosis of homozygotes/heterozygotes compared to wildtypes, 792 respectively using Wilcoxon rank tests. For few compound heterozygous variants (as 793 indicated in the manuscript), we also performed survival analyses using age at first disease 794 diagnosis as outcome using a Cox proportional hazard model with the same covariates 795 that were also used in the GWAS (sex, age, genotyping batch, first 10 principal 796 components). 


\section{References}

800

8011 Abifadel, M. et al. Mutations in PCSK9 cause autosomal dominant 802 hypercholesterolemia. Nat Genet 34, 154-156, doi:10.1038/ng1161 (2003).

8032 Bell, D. W. et al. Heterozygous germ line hCHK2 mutations in Li-Fraumeni 804 syndrome. Science 286, 2528-2531, doi:10.1126/science.286.5449.2528 805 806 807 (1999).

3 Zuk, O. et al. Searching for missing heritability: designing rare variant association studies. Proc Natl Acad Sci $U S A$ 111, E455-464, doi:10.1073/pnas.1322563111 (2014).

4 Martin, H. C. et al. Quantifying the contribution of recessive coding variation to developmental disorders. Science 362, 1161-1164, doi:10.1126/science.aar6731 (2018).

5 Diaz, G. A. et al. Gaucher disease: the origins of the Ashkenazi Jewish N370S and 84GG acid beta-glucosidase mutations. Am J Hum Genet 66, 1821-1832, doi:10.1086/302946 (2000).

6 Moises, H. W. et al. An international two-stage genome-wide search for schizophrenia susceptibility genes. Nat Genet 11, 321-324, doi:10.1038/ng1195-321 (1995).

819

820

821

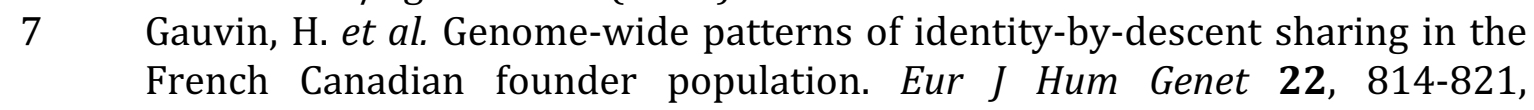
doi:10.1038/ejhg.2013.227 (2014).

8 Puffenberger, E. G. et al. A missense mutation of the endothelin-B receptor gene in multigenic Hirschsprung's disease. Cell 79, 1257-1266, doi:10.1016/00928674(94)90016-7 (1994).

9 Belbin, G. M. et al. Genetic identification of a common collagen disease in puerto ricans via identity-by-descent mapping in a health system. Elife 6, doi:10.7554/eLife.25060 (2017).

10 Peltonen, L., Jalanko, A. \& Varilo, T. Molecular genetics of the Finnish disease heritage. Hum Mol Genet 8, 1913-1923, doi:10.1093/hmg/8.10.1913 (1999).

830

831

832

833

834

835

11 Peltonen, L., Palotie, A. \& Lange, K. Use of population isolates for mapping complex traits. Nat Rev Genet 1, 182-190, doi:10.1038/35042049 (2000).

12 Kerminen, S. et al. Fine-Scale Genetic Structure in Finland. G3 (Bethesda) 7, 3459-3468, doi:10.1534/g3.117.300217 (2017).

13 Jakkula, E. et al. The genome-wide patterns of variation expose significant substructure in a founder population. Am J Hum Genet 83, 787-794, doi:10.1016/j.ajhg.2008.11.005 (2008).

14 Martin, A. R. et al. Haplotype Sharing Provides Insights into Fine-Scale Population History and Disease in Finland. Am J Hum Genet 102, 760-775, doi:10.1016/j.ajhg.2018.03.003 (2018).

15 Fuchshuber, A. et al. Presymptomatic diagnosis of familial steroid-resistant nephrotic syndrome. Lancet 347, 1050-1051, doi:10.1016/s01406736(96)90193-2 (1996).

16 Herva, R., Tyynela, J., Hirvasniemi, A., Syrjakallio-Ylitalo, M. \& Haltia, M. Northern epilepsy: a novel form of neuronal ceroid-lipofuscinosis. Brain Pathol 10, 215-222, doi:10.1111/j.1750-3639.2000.tb00255.x (2000). 
next-generation sequencing era. Hum Mutat 34, 1458-1466, doi:10.1002/humu.22389 (2013).

18 Locke, A. E. et al. Exome sequencing of Finnish isolates enhances rare-variant association power. Nature 572, 323-328, doi:10.1038/s41586-019-1457-z (2019).

19 Lim, E. T. et al. Distribution and medical impact of loss-of-function variants in the Finnish founder population. PLoS Genet 10, e1004494, doi:10.1371/journal.pgen.1004494 (2014).

856

857

858

859

860

861

862

863

864

865

866

867

868

869

870

871

872

873

874

875

876

877

878

879

880

881

882

883

884

885

886

887

888

889

890

891

892

893

20 Ostrer, H. A genetic profile of contemporary Jewish populations. Nat Rev Genet 2, 891-898, doi:10.1038/35098506 (2001).

21 Wildenberg, S. C. et al. A gene causing Hermansky-Pudlak syndrome in a Puerto Rican population maps to chromosome 10q2. Am J Hum Genet 57, 755-765 (1995).

22 Bouchard, J. P., Barbeau, A., Bouchard, R. \& Bouchard, R. W. Autosomal recessive spastic ataxia of Charlevoix-Saguenay. Can J Neurol Sci 5, 61-69 (1978).

23 A novel gene containing a trinucleotide repeat that is expanded and unstable on Huntington's disease chromosomes. The Huntington's Disease Collaborative Research Group. Cell 72, 971-983, doi:10.1016/00928674(93)90585-e (1993).

24 Wang, G., Sarkar, A., Carbonetto, P. \& Stephens, M. A simple new approach to variable selection in regression, with application to genetic fine mapping. Journal of the Royal Statistical Society: Series B (Statistical Methodology) 82, 1273-1300, doi:https://doi.org/10.1111/rssb.12388 (2020).

25 Kinnunen, S. et al. Spectrum of mutations in CFTR in Finland: 18 years followup study and identification of two novel mutations. J Cyst Fibros 4, 233-237, doi:10.1016/j.jcf.2005.06.002 (2005).

26 Kals, M. et al. Advantages of genotype imputation with ethnically matched reference panel for rare variant association analyses. bioRxiv, 579201, doi:10.1101/579201 (2019).

27 Zhou, W. et al. Efficiently controlling for case-control imbalance and sample relatedness in large-scale genetic association studies. Nat Genet 50, 13351341, doi:10.1038/s41588-018-0184-y (2018).

28 Landrum, M. J. et al. ClinVar: public archive of interpretations of clinically relevant variants. Nucleic Acids Res 44, D862-868, doi:10.1093/nar/gkv1222 (2016).

29 Karczewski, K. J. et al. The mutational constraint spectrum quantified from variation in 141,456 humans. Nature 581, 434-443, doi:10.1038/s41586-0202308-7 (2020).

30 Richards, S. et al. Standards and guidelines for the interpretation of sequence variants: a joint consensus recommendation of the American College of Medical Genetics and Genomics and the Association for Molecular Pathology. Genet Med 17, 405-424, doi:10.1038/gim.2015.30 (2015).

31 Niroula, A. \& Vihinen, M. How good are pathogenicity predictors in detecting benign variants? PLoS Comput Biol 15, e1006481, doi:10.1371/journal.pcbi.1006481 (2019).

32 Robertson, D. et al. Isolated failure of autonomic noradrenergic neurotransmission. Evidence for impaired beta-hydroxylation of dopamine. 
The New England journal of medicine 314, 1494-1497, doi:10.1056/NEJM198606053142307 (1986).

33 Tanaka, K. et al. Analysis of a human DNA excision repair gene involved in group A xeroderma pigmentosum and containing a zinc-finger domain. Nature 348, 73-76, doi:10.1038/348073a0 (1990).

34 Hirai, Y. et al. Increased risk of skin cancer in Japanese heterozygotes of xeroderma pigmentosum group A. J Hum Genet 63, 1181-1184, doi:10.1038/s10038-018-0495-y (2018).

35 Benson, D. W. et al. Congenital sick sinus syndrome caused by recessive mutations in the cardiac sodium channel gene (SCN5A). J Clin Invest 112, 10191028, doi:10.1172/JCI18062 (2003).

36 Bycroft, C. et al. The UK Biobank resource with deep phenotyping and genomic data. Nature 562, 203-209, doi:10.1038/s41586-018-0579-z (2018).

37 Ivarsdottir, E. V. et al. The genetic architecture of age-related hearing impairment revealed by genome-wide association analysis. Commun Biol 4, 706, doi:10.1038/s42003-021-02224-9 (2021).

38 Lin, S. H., Brown, D. W. \& Machiela, M. J. LDtrait: An Online Tool for Identifying Published Phenotype Associations in Linkage Disequilibrium. Cancer Res 80, 3443-3446, doi:10.1158/0008-5472.CAN-20-0985 (2020).

39 Adank, M. A. et al. CHEK2*1100delC homozygosity is associated with a high breast cancer risk in women. J Med Genet 48, 860-863, doi:10.1136/jmedgenet-2011-100380 (2011).

40 Nathan, V. et al. Germline variants in oculocutaneous albinism genes and predisposition to familial cutaneous melanoma. Pigment Cell Melanoma Res 32, 854-863, doi:10.1111/pcmr.12804 (2019).

41 Tagliabue, E. et al. MC1R gene variants and non-melanoma skin cancer: a pooled-analysis from the M-SKIP project. Br J Cancer 113, 354-363, doi:10.1038/bjc.2015.231 (2015).

42 Baxter, E. J. et al. Acquired mutation of the tyrosine kinase JAK2 in human myeloproliferative disorders. Lancet 365, 1054-1061, doi:10.1016/S01406736(05)71142-9 (2005).

43 Byrnes, A. M. et al. Mutations in GDF5 presenting as semidominant brachydactyly A1. Hum Mutat 31, 1155-1162, doi:10.1002/humu.21338 (2010).

44 Wilkie, A. O. Dominance and Recessivity. eLS (2006). $<$ https://onlinelibrary.wiley.com/doi/abs/10.1038/npg.els.0005475>.

45 Birchler, J. A. \& Veitia, R. A. Gene balance hypothesis: connecting issues of dosage sensitivity across biological disciplines. Proc Natl Acad Sci U S A 109, 14746-14753, doi:10.1073/pnas.1207726109 (2012).

46 Amorim, C. E. G. et al. The population genetics of human disease: The case of recessive, lethal mutations. PLoS Genet 13, e1006915, doi:10.1371/journal.pgen.1006915 (2017).

47 Burkhart, B. D., Montgomery, E., Langley, C. H. \& Voelker, R. A. Characterization of Allozyme Null and Low Activity Alleles from Two Natural Populations of DROSOPHILA MELANOGASTER. Genetics 107, 295-306 (1984).

48 Wright, C. F. et al. Assessing the Pathogenicity, Penetrance, and Expressivity of Putative Disease-Causing Variants in a Population Setting. Am J Hum Genet 104, 275-286, doi:10.1016/j.ajhg.2018.12.015 (2019). 
49 Caridi, G. et al. Clinical features and long-term outcome of nephrotic syndrome associated with heterozygous NPHS1 and NPHS2 mutations. Clin J Am Soc Nephrol 4, 1065-1072, doi:10.2215/CJN.03910808 (2009).

50 Crystal, R. G. Alpha 1-antitrypsin deficiency, emphysema, and liver disease. Genetic basis and strategies for therapy. J Clin Invest 85, 1343-1352, doi:10.1172/JCI114578 (1990).

950

951

952

953

954

955

956

957

958

959

960

961

962

963

964

965

966

967

968

969

970

971

972

973

974

975

976

51 Chan, D. K. \& Chang, K. W. GJB2-associated hearing loss: systematic review of worldwide prevalence, genotype, and auditory phenotype. Laryngoscope 124, E34-53, doi:10.1002/lary.24332 (2014).

52 Vogel, F. Clinical consequences of heterozygosity for autosomal-recessive diseases. Clin Genet 25, 381-415 (1984).

53 Gouagna, L. C. et al. Genetic variation in human HBB is associated with Plasmodium falciparum transmission. Nat Genet 42, 328-331, doi:10.1038/ng.554 (2010).

54 Pier, G. B. et al. Salmonella typhi uses CFTR to enter intestinal epithelial cells. Nature 393, 79-82, doi:10.1038/30006 (1998).

55 Ayi, K., Turrini, F., Piga, A. \& Arese, P. Enhanced phagocytosis of ringparasitized mutant erythrocytes: a common mechanism that may explain protection against falciparum malaria in sickle trait and beta-thalassemia trait. Blood 104, 3364-3371, doi:10.1182/blood-2003-11-3820 (2004).

56 Butters, T. D. et al. Mechanistic links between Na+ channel (SCN5A) mutations and impaired cardiac pacemaking in sick sinus syndrome. Circ Res 107, 126137, doi:10.1161/CIRCRESAHA.110.219949 (2010).

57 Grubb, R. Correlation between Lewis blood group and secretor character in man. Nature 162, 933, doi:10.1038/162933a0 (1948).

58 Belbin, G. M. et al. Towards a fine-scale population health monitoring system. bioRxiv, 780668, doi:10.1101/780668 (2019).

59 Gudbjartsson, D. F. et al. A frameshift deletion in the sarcomere gene MYL4 causes early-onset familial atrial fibrillation. Eur Heart $J$ 38, 27-34, doi:10.1093/eurheartj/ehw379 (2017).

60 Guindo-Martinez, M. et al. The impact of non-additive genetic associations on age-related complex diseases. Nat Commun 12, 2436, doi:10.1038/s41467021-21952-4 (2021). 